\title{
Nanocrystalline iron at high pressure
}

\author{
B. Chen, ${ }^{a}$ D. Penwell, and M. B. Kruger
}

Department of Physics, University of Missouri, Kansas City, Missouri 64110

\author{
A. F. Yue and B. Fultz \\ Department of Engineering and Applied Science, California Institute of Technology, Pasadena, \\ California 91125
}

(Received 4 January 2001; accepted for publication 29 January 2001)

\begin{abstract}
$\mathrm{X}$-ray diffraction measurements were performed on nanocrystalline iron up to $46 \mathrm{GPa}$. For nanocrystalline $\epsilon$-Fe, analysis of lattice parameter data provides a bulk modulus, $K$, of 179 $\pm 8 \mathrm{GPa}$ and a pressure derivative of the bulk modulus, $K^{\prime}$, of $3.6 \pm 0.7$, similar to the large-grained control sample. The extrapolated zero-pressure unit cell volume of nanocrystalline $\epsilon$-Fe is 22.9 $\pm 0.2 \AA^{3}$, compared to $22.3 \pm 0.2 \AA^{3}$ for large-grained $\epsilon$-Fe. No significant grain growth was observed to occur under pressure. (C) 2001 American Institute of Physics.
\end{abstract}

[DOI: $10.1063 / 1.1357780]$

\section{INTRODUCTION}

The properties of many materials depend on crystallite size when the size is less than $100 \mathrm{~nm}$, generating a tremendous amount of interest in the properties of nanocrystalline materials and in methods for their synthesis. ${ }^{1}$ Most highpressure research on nanocrystalline materials has been on semiconductors, although some work on insulators and metals has been reported recently. In general it has been found that nanocrystals undergo the same phase transitions as their large-grained counterparts, although at elevated pressures. ${ }^{2-4}$ Differences in the phase diagrams of nanocrystals and their large-grained counterparts have been used to argue for the possibility of synthesizing new structures using nanocrystalline starting materials, as has been realized recently with nanocrystalline alumina. ${ }^{5}$ In addition, for some materials there is evidence that the bulk modulus and equation of state depend on crystallite size. ${ }^{5,6}$

The Young's modulus of some nanocrystalline materials may be a function of particle size for reasons of porosity. ${ }^{7}$ On the other hand, a Mössbauer spectrometry study on nanocrystalline iron $(\alpha-\mathrm{Fe})$ has indicated that the grain boundary regions have a bulk modulus approximately $15 \%$ of that of the interior crystalline regions. ${ }^{8}$ A simulation of nanophase silicon has suggested that the bulk modulus of $n$-Si is smaller than that of bulk $\mathrm{Si}$, although the reason is not clear. ${ }^{9}$ In contrast, embedded atom method calculations predicted a decrease in interatomic distances in nanocrystalline materials as compared with the large-grained metals, implying an enhanced bulk modulus for these nanocrystals. ${ }^{10}$

Nanocrystalline iron $(n-\mathrm{Fe})$ has been the subject of many experimental and theoretical studies. Investigations on $n$-Fe have reported magnetic, mechanical, thermal, and electrical properties, surface passivation, and mechanical properties such as ductility, hardness, and phonon density-of-states. ${ }^{11-15}$ Here we report the results of high pressure $\mathrm{x}$-ray diffraction experiments, performed to measure

${ }^{a)}$ Electronic mail: bcdb7@umkc.edu the equation of state of $n$-Fe. We report a bulk modulus for the crystallites in nanocrystalline hexagonal $\epsilon$-Fe that differs little from the bulk modulus of large-grained material, although there seems to be a larger unit cell volume for $n$-Fe.

\section{EXPERIMENT}

Nanocrystalline iron was made by mechanical attrition of Fe of $99.995 \%$ purity. The material was milled in a Spex $8000 \mathrm{mixer} / \mathrm{mill}$ with a hardened steel vial, lid, and balls and a ball-to-powder weight ratio of 20:1. The vial and lid had knife edges machined into them, which were used to make a metal-metal seal against a copper gasket. X-ray diffraction line shapes were analyzed with a $\Delta k$ vs $k$ extrapolation method to determine the average crystallite size to be $10 \mathrm{~nm}$. The powders were studied before and after compaction with a Philips EM420 transmission electron microscope operated at $120 \mathrm{keV}$. Dark-field images showed that the crystallite sizes were consistent with the size of $10 \mathrm{~nm}$ obtained from $\mathrm{x}$-ray line shapes. For comparison, a control sample was prepared from some of the same powder by annealing for $0.5 \mathrm{~h}$ at $700^{\circ} \mathrm{C}$ in an evacuated quartz ampoule and cooled at a rate of $50^{\circ} \mathrm{C} / \mathrm{h}$. This resulted in a grain size larger than 50 $\mathrm{nm}$, the upper limit of our methods for measurement of crystallite size.

For the high-pressure x-ray diffraction (XRD) experiments, the powder samples were loaded into a Mao-Bell type diamond cell with anvils having $350 \mu \mathrm{m}$ culets. ${ }^{16} \mathrm{~A}$ spring-steel gasket, with a chamber having a diameter of 160 $\mu \mathrm{m}$, was used to contain the sample. A mixture of 4:1 methanol:ethanol was also loaded into the sample compartment to reduce the nonhydrostatic stresses. In addition, a small amount of gold powder $(<2 \%)$ was included to determine the pressure, using the equation of state of gold. ${ }^{17} \mathrm{X}$-ray diffraction data were measured from 0 to $46 \mathrm{GPa}$ under quasihydrostatic conditions, using $\mathrm{x}$-ray diffraction at beamline X17C of the National Synchrotron Light Source. For comparison, $\mathrm{x}$-ray diffraction patterns from the control sample of annealed powder were measured under similar 


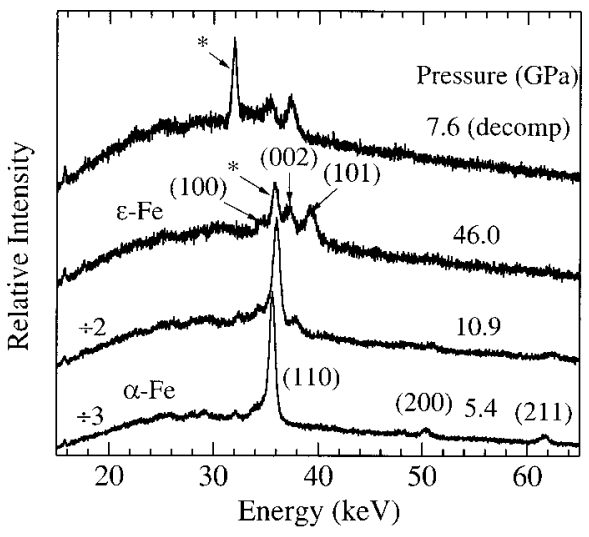

FIG. 1. Representative diffraction patterns at 5.4, 10.9, 46.0, and 7.6 GPa of quasihydrostatically compressed $n$-Fe. The pattern taken at $7.6 \mathrm{GPa}$ is on decompression. The peaks labeled with asterisk are (311) of iron oxide.

conditions to $55 \mathrm{GPa}$, at beamline 2-2 of the Stanford Synchrotron Radiation Laboratory. The diffraction data at both beamlines were acquired in energy dispersive mode, using liquid nitrogen cooled Ge detectors. At X17C the detector was positioned at $2 \theta=9.958^{\circ} \pm 0.002^{\circ}$ and at beamline $2-2$ at $2 \theta=15.272^{\circ} \pm 0.013^{\circ}$. All data were acquired at $\sim 295^{\circ} \mathrm{K}$. An intense peak of iron oxide $\left(\mathrm{Fe}_{2} \mathrm{O}_{3}\right)$ was also observed in some of the diffraction patterns. The oxide is a passivation layer confined to the surface of the powder particles.

\section{RESULTS AND DISCUSSION}

The transformation pressures for the $\alpha-\epsilon$ and $\epsilon-\alpha$ transitions of large-grained Fe have been well studied, using a variety of pressure media. ${ }^{18}$ Using 4:1 methanol:ethanol as the pressure transmitting medium for large-grained $\mathrm{Fe}$, the $\alpha-\epsilon$ transition begins at $14.3 \mathrm{GPa}$ and is complete at 17.5 $\mathrm{GPa}$, while on decompression, $\epsilon$-Fe starts to convert back to $\alpha$-Fe at $11.9 \mathrm{GPa}$ and the transformation is complete by 7 $\mathrm{GPa}^{18}$

The XRD patterns showed that nanocrystalline $\alpha$-Fe began transforming to $\epsilon$-Fe when it was compressed to 10.9

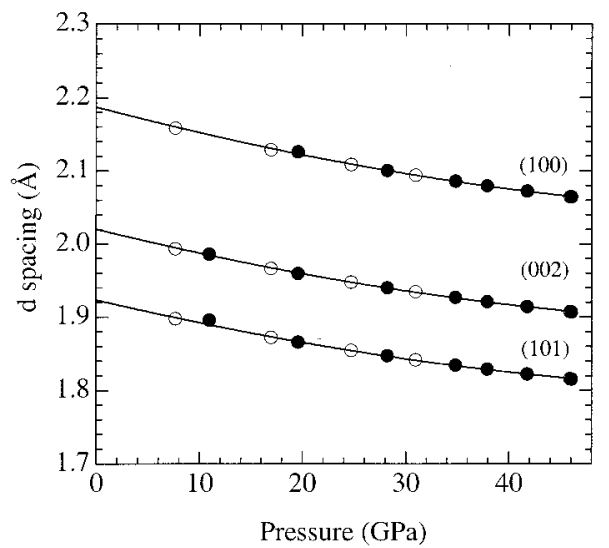

FIG. 2. Interplanar $d$ spacing of $n$-Fe as a function of pressure for quasihydrostatic compression. The closed symbols indicate data taken upon compression while the open symbols represent data taken upon decompression. The solid lines are guides.

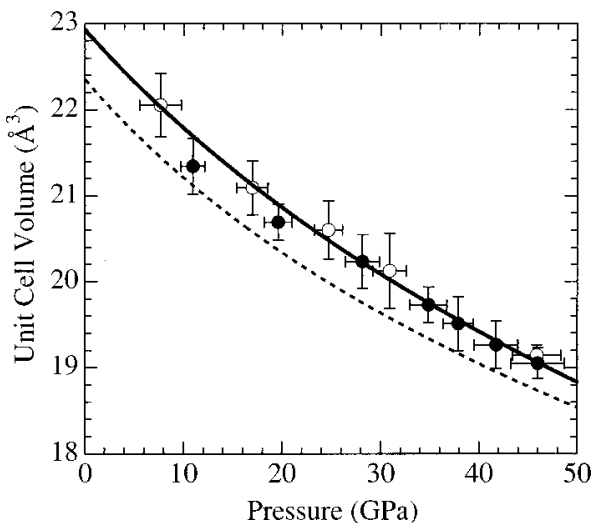

FIG. 3. Pressure dependence of the unit cell volume for $n$-Fe. The closed symbols represent experimental data for quasihydrostatic compression, while the open symbols represent data taken upon decompression. The solid line is the best fit of the Birch-Murnaghan equation of state to the quasihydrostatic compression data. The dashed line is the Birch-Murnaghan equation of state of large-grained Fe (see Ref. 22).

GPa. The $\epsilon$ phase remained to $46 \mathrm{GPa}$, the highest pressure of this study. Upon decompression, $\epsilon$-Fe did not transform back to the $\alpha$ phase at 7.6 $\mathrm{GPa}$, which was the lowest pressure measured in situ. Transmission electron microscopy showed that no $\epsilon$-Fe remained at $0 \mathrm{GPa}$, however. These images also showed that the crystallite size was not changed significantly after compaction.

The 100, 002, and $101 \mathrm{x}$-ray diffraction lines were obtained at each pressure for $\epsilon$-Fe (Fig. 1). The pressure dependencies of the $d$ spacing and the unit cell volume of $n$-Fe from 8 to $46 \mathrm{GPa}$ are summarized in Figs. 2 and 3. We determined the lattice parameters of $n$-Fe at each pressure, using a weighted average of the $d$ spacing, and analyzed the data in terms of the Birch-Murnaghan (Eulerian finite strain) equation of state ${ }^{19}$

$$
F_{V}=K\left[1-1.5(4-K) f_{V}\right],
$$

where the negative of the Eulerian strain measure $f_{V}$ and normalized pressure $F_{V}$ are defined as

$$
f_{V}=0.5\left[\left(\frac{V}{V_{0}}\right)^{-2 / 3}-1\right],
$$

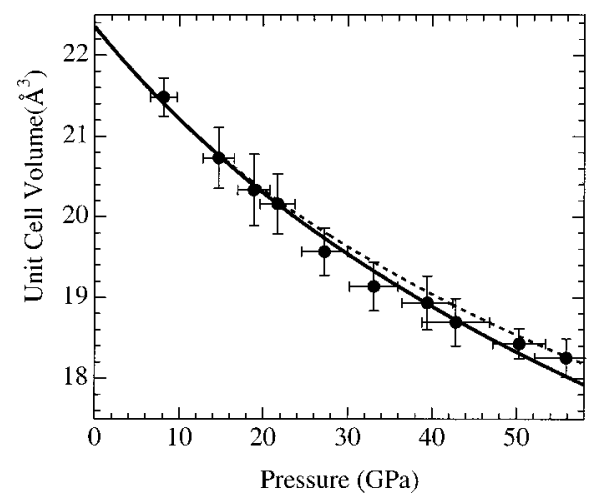

FIG. 4. Pressure dependence of the unit cell volume for annealed $n$-Fe. All data was taken on compression. The solid line is the best fit of the BirchMurnaghan equation of state to the data. The dashed line is the BirchMurnaghan equation of state of large-grained Fe (see Ref. 22). 


$$
F_{V}=P\left[3 f_{V}\left(1+2 f_{V}\right)^{2.5}\right]^{-1},
$$

with $V_{0}$ being unit cell volume at zero pressure.

For the case of $\epsilon$-Fe, the unit cell volume at zero pressure is not directly measurable. We obtained it by extrapolation using the modified Birch-Murnaghan equation of state ${ }^{20}$

$$
G=a+b g+c g^{2}+d g^{3}+\cdots,
$$

where the effective strain, $g$, and new normalized pressure, $G$, are defined as

$$
\begin{aligned}
& g=0.5\left[\left(\frac{V}{V^{*}}\right)^{-2 / / 3}-1\right], \\
& G=P\left[3(1+2 g)^{2.5}\right]^{-1},
\end{aligned}
$$

where $V^{*}$ is an arbitrary reference unit cell volume. A linear fit to Eq. (4) yields $g_{0}$ such that $G\left(g_{0}\right)=0$. The unit cell volume at zero pressure, $V_{0}$, is

$$
V_{0}=V^{*}\left(1+2 g_{0}\right)^{-1.5} \text {. }
$$

The $G-g$ analysis gives $V_{0}$ of nanocrystalline $\epsilon$-Fe as 22.9 $\pm 0.2 \AA^{3}$. The slight increase of the unit cell volume of $n$-Fe over $V_{0}$ of large-grained $\epsilon$ - $\mathrm{Fe}\left(22.3 \pm 0.2 \AA^{3}\right)$ may be accounted for by lattice relaxation of $n-\mathrm{Fe}^{21}$

The intercept and slope of the $P-V$ data, recast into the form $F-f$, give the bulk modulus $K$ and its pressure derivative $K^{\prime}$ at zero pressure. Fits to the data yield $K=179.4$ $\pm 8.1 \mathrm{GPa}$, and $K^{\prime}=3.57 \pm 0.65$ for quasihydrostatically compressed $n$-Fe of the $\epsilon$ phase. For comparison, quasihydrostatic XRD patterns were collected from 3.4 to $55 \mathrm{GPa}$ for our control sample of annealed powder, giving unit cell volumes shown in Fig. 4. The $G-g$ and $F-f$ analysis gives $V_{0}=22.2 \pm 0.2 \AA^{3}, \quad K=173.1 \pm 9.7 \mathrm{GPa}, \quad$ and $\quad K^{\prime}=3.79$ \pm 0.75 for our control sample. A Birch-Murnaghan fit to data from a previous study on large-grained $\epsilon$-Fe yields a bulk modulus of $165 \pm 4 \mathrm{GPa}$ with a $K^{\prime}=5.33(9)$-this curve is included in Figs. 3 and $4 .{ }^{22}$ Within experimental uncertainty, the equations of state are not different for $n$-Fe, our control sample of annealed $n-\mathrm{Fe}$, and prior results on large-grained $\epsilon$-Fe.

A previous study on the influence of temperature and compaction pressure on the grain size of $n$-Fe reported grain growth during thermal annealing, but no effect from compaction pressure up to $\sim 3 \mathrm{GPa} .{ }^{14}$ We used transmission electron microscopy to check the grain size of quenched $n$-Fe after compaction to $40 \mathrm{GPa}$ and found no significant grain growth. The measured $\mathrm{x}$-ray line shapes also showed no significant changes after compaction.

Our measurements were performed with modest increments in pressure, so the pressures of the $\alpha \rightarrow \epsilon$ and the $\epsilon$ $\rightarrow \alpha$ transitions were not obtained with precision. There is some evidence that these phase transitions both occur at lower pressures for $n$-Fe, but this is a subject of ongoing study.

\section{CONCLUSIONS}

We performed synchrotron $\mathrm{x}$-ray diffraction on nanocrystalline $\epsilon$-Fe in a diamond-anvil cell to quasihydrostatic pressures of $46 \mathrm{GPa}$. A fit of the diffraction data to the Birch-Murnaghan equation of state yields a bulk modulus for the crystallites of nanocrystalline $\epsilon$-Fe of $K=179.4$ $\pm 8.1 \mathrm{GPa}$ with $K^{\prime}=3.57 \pm 0.65$, similar to that of largegrained $\epsilon$-Fe. However, the extrapolated initial unit cell volume of nanocrystalline $\epsilon$-Fe at room pressure, $V_{0}=22.9$ $\pm 0.2 \AA^{3}$, is larger than the $22.3 \pm 0.2 \AA^{3}$ for large-grained $\epsilon$-Fe, suggesting lattice relaxation in nanocrystalline materials. No significant grain growth was observed to occur under pressure.

\section{ACKNOWLEDGMENTS}

The authors thank Dr. Jingzhu Hu for help with the XRD operation in X17C, NSLS. This work was supported by the National Science Foundation and the Petroleum Research Foundation.

${ }^{1}$ A. S. Edelstein and R. C. Cammarata, Institute of Physics (IOP, Bristol, 1996).

${ }^{2}$ S. H. Tolbert and A. P. Alivisatos, Science 265, 373 (1994).

${ }^{3}$ S. H. Tolbert, A. B. Herhold, L. E. Brus, and A. P. Alivisatos, Phys. Rev. Lett. 76, 4384 (1996).

${ }^{4}$ C. C. Chen, A. B. Herhold, C. S. Johnson, and A. P. Alivisatos, Science 276, 398 (1997).

${ }^{5}$ B. Chen, D. Penwell, L. R. Benedetti, W. Caldwell, R. Jeanloz, and M. B. Kruger, Bull. Am. Phys. Soc. 44, 112 (1999).

${ }^{6}$ S. H. Tolbert and A. P. Alivisatos, Annu. Rev. Phys. Chem. 46, 595 (1995)

${ }^{7}$ G. E. Fougere, L. Riester, M. Ferber, J. R. Weertman, and R. W. Siegel, Mater. Sci. Eng., A 204, 1 (1995).

${ }^{8}$ S. Trapp, C. T. Liimbach, U. Gonser, S. J. Campbell, and H. Gleiter, Phys. Rev. Lett. 75, 3760 (1995).

${ }^{9}$ J. A. Lupo and M. J. Sabochic, Nanostruct. Mater. 1, 131 (1992).

${ }^{10}$ A. Kara and T. S. Rahman, Phys. Rev. Lett. 81, 1453 (1998).

${ }^{11}$ C.-M. Hsu, H.-M. Lin, K.-R. Tsai, and P.-Y. Lee, J. Appl. Phys. 76, 4793 (1994)

${ }^{12}$ H. Y. Bai, L. L. Luo, D. Jin, and J. R. Sun, J. Appl. Phys. 79, 361 (1996).

${ }^{13}$ W. Gong, H. Li, Z. Zhao, and J. Chen, J. Appl. Phys. 69, 5119 (1991).

${ }^{14}$ T. R. Malow and C. C. Koch, Metall. Mater. Trans. A 29A, 2285 (1998).

${ }^{15}$ B. Fultz, J. L. Robertson, T. A. Stephens, L. J. Nagel, and S. Spooner, J. Appl. Phys. 79, 8318 (1996).

${ }^{16}$ H. K. Mao, P. M. Bell, K. J. Dunn, R. M. Chrenko, and R. C. DeVries, Rev. Sci. Instrum. 50, 1002 (1979).

${ }^{17}$ D. L. Heinz and R. Jeanloz, J. Appl. Phys. 55, 885 (1984).

${ }^{18}$ R. Boehler, N. Vonbargen, and A. Chopelas, J. Geophys. Res. B 95, 21731 (1990).

${ }^{19}$ F. Birch, J. Geophys. Res. B 83, 1257 (1978).

${ }^{20}$ R. Jeanloz, Geophys. Res. Lett. 8, 1219 (1981).

${ }^{21}$ H.-K. Mao, W. A. Bassett, and T. Takahashi, J. Appl. Phys. 38, 272 (1967).

${ }^{22}$ H. K. Mao, Y. Wu, L. C. Chen, and J. F. Shu, J. Geophys. Res. B 95, 21737 (1990). 